Annales Geophysicae (2002) 20: 311-320 (c) European Geophysical Society 2002

\title{
Cusp-like plasma in high altitudes: a statistical study of the width and location of the cusp from Magion-4
}

\author{
J. Měrka, J. Šafránková, and Z. Němeček \\ Charles University, Faculty of Mathematics and Physics, V Holesovickach 2, 18000 Praha 8, Czech Republic
}

Received: 15 January 2001 - Revised: 25 June 2001 - Accepted: 23 August 2001

\begin{abstract}
The width of the cusp region is an indicator of the strength of the merging process and the degree of opening of the magnetosphere. During three years, the Magion-4 satellite, as part of the Interball project, has collected a unique data set of cusp-like plasma observations in middle and high altitudes. For a comparison of high- and low-altitude cusp determination, we map our observations of cusp-like plasma along the magnetic field lines down to the Earth's surface. We use the Tsyganenko and Stern 1996 model of the magnetospheric magnetic field for the mapping, taking actual solar wind and IMF parameters from the Wind observations. The footprint positions show substantial latitudinal dependence on the dipole tilt angle. We fit this dependence with a linear function and subtract this function from observed cusp position. This process allows us to study both statistical width and location of the inspected region as a function of the solar wind and IMF parameters.

Our processing of the Magion-4 measurements shows that high-altitude regions occupied by the cusp-like plasma (cusp and cleft) are projected onto a much broader area (in magnetic local time as well as in a latitude) than that determined in low altitudes. The trends of the shift of the cusp position with changes in the IMF direction established by low-altitude observations have been confirmed.
\end{abstract}

Key words. Magnetospheric physics (magnetopause, cusp and boundary layer; solar wind - magnetosphere interactions)

\section{Introduction}

The cusp region and high-latitude boundary layers are generally recognized as being key regions for the solar windmagnetosheath coupling, especially in critical ranges of the high-altitude cusp for large radial distances $\left(>8 R_{E}\right)$. Highlatitude observations at middle and high altitudes have been restricted to a few satellites with appropriate orbits, such

Correspondence to: J. Měrka (jan.merka@mff.cuni.cz) as HEOS-2 (Paschmann et al., 1978), Prognoz-7 (Lundin, 1985), Hawkeye (Farrell and Van Allen, 1990), and at present, Polar (Zhou et al., 1999).

\subsection{Cusp definition and its average location}

On the other hand, a tremendous volume of cusp observations have been accumulated by low-altitude polarorbiting satellites which provide longitudinal or latitudinal cuts through the cusp. This investigation has been extensively provided by the Defense Meteorological Satellite Program (DMSP). Using $\sim 12000$ crossings, Newell and Meng (1988) developed a simple and practical definition of the cusp and cleft/LLBL regions. If $E_{e}<200 \mathrm{eV}, E_{i}<2700 \mathrm{eV}$, $j_{i}>10^{10} \mathrm{eV} / \mathrm{cm}^{2}$.s.sr, and $j_{e}>6 \times 10^{10} \mathrm{eV} / \mathrm{cm}^{2} . s . s r$, the region is called the cusp $\left(E_{i}\right.$ and $E_{e}$ are the average ion and electron energies, $j_{i}$ and $j_{e}$ are the energy fluxes of ions and electrons, respectively). If either $3000 \mathrm{eV}<E_{i}<6000 \mathrm{eV}$ or $220 \mathrm{eV}<E_{e}<600 \mathrm{eV}$, the region was identified as cleft/LLBL. Statistical processing of the DMSP data has shown that the mean ionospheric projection of both cusp and cleft regions is located between $75.3^{\circ}$ and $76.8^{\circ}$ of magnetic latitude (MLAT) (Newell and Meng, 1989).

In middle altitudes, Kremser and Lundin (1990) found the cusp-like plasma in broader ranges: $\sim 75.5^{\circ}-83.5^{\circ}$ of invariant latitudes (INL) and $8-15 \mathrm{~h}$ of magnetic local time (MLT) based on the Viking data $(11000 \mathrm{~km}-13500 \mathrm{~km}$ of altitude). Their criteria for the classification of different cusp regions included the presence or absence of ions and electrons of the magnetosheath origin, and the presence/absence of acceleration signatures on electron fluxes. The classification is based on the visual inspection of the ion and electron energy spectra. They compared their observations with lowaltitude statistical studies (Newell and Meng, 1988) and they found that there is good but not full agreement between the low- and middle-altitude structures of the cusp/cleft region. The disagreement may be partly due to the different data sets and partly due to slightly different definitions of particular regions. 
Due to the highly eccentric orbit of the Hawkeye satellite with apogee at $\sim 21 R_{E}$, Eastman et al. (2000) could perform a statistical study of the cusp near the magnetopause and they found that the median and mean invariant latitudes of the cusp are both $\Lambda=79.9^{\circ} \pm 1.9^{\circ}$. Their criteria for cusp identification included a combination of the following signatures: a mixture of magnetosheath and magnetospheric plasma, "DC" magnetic field depression, lowfrequency, broadband magnetic noise from $1 \mathrm{~Hz}$ up to ion cyclotron frequency and enhanced broadband electrostatic noise. Both Hawkeye's cusp observations (Eastman et al., 2000) and the new magnetopause model of Boardsen et al. (2000) showed that the cusp indentation appears to deepen and spread out in latitude with increasing dipole tilt angles. Thus, as the dipole tilts more towards the oncoming magnetosheath flow, the indentation becomes enlarged and the cusp outflow region, the plasma mantle, becomes more flared out relative to the Earth-Sun line. In contrast, as the dipole tilts away from the Sun, the cusp indentation is reduced and the cusp outflow region becomes closer to the boundary observed at low-latitudes.

Zhou and Russell (1997) used magnetopause crossings observed by Hawkeye to show that the cusp high-altitude position is affected by the dipole tilt angle and demonstrate that in the neighborhood of the magnetopause, the polar cusp location is controlled by the tilt of the dipole.

The Polar spacecraft observed the cusp region at high altitudes of 4.8-8.8 $R_{E}$ in the Northern Hemisphere. The criteria used by Zhou et al. (1999) to identify the cusp crossings are: a depression of the magnetic field strength below the neighboring background level greater than $1 \mathrm{nT}$, a sudden increase in the low-energy ion and electron densities to a value greater than $5 \mathrm{~cm}^{-3}$, an electron thermal energy less than $100 \mathrm{eV}$, and a presence of a significant portion of $\mathrm{He}^{++}$, which indicates the solar wind origin of plasma. In a recent paper, Zhou et al. (1999) demonstrated the dipole tilt angle effect on the cusp location using a new data set from Polar. They concluded that the position of the cusp in investigated altitudes depends significantly on the dipole tilt angle. When the dipole tilts more toward the Sun, the cusp moves more poleward to higher invariant latitudes, from $77.2^{\circ}$ at $-30^{\circ}$ tilt, to $80.0^{\circ}$ at $0^{\circ}$ tilt, to $81.8^{\circ}$ at $30^{\circ}$, or roughly $1^{\circ}$ for every $14^{\circ}$ of tilt. They noted that the behaviour of the invariant latitude of the cusp, as seen by Polar, is entirely consistent with that seen at low altitudes (Newell and Meng (1989), showed that the cusp moves $1^{\circ}$ in magnetic latitude for a $17^{\circ}$ increase in the tilt angle).

A comparison of these two results suggests an increasing shift in the cusp projection with the altitude. Indeed, Němeček et al. (2000), who analyzed Magion-4 cusp observations near the magnetopause $\left(\leq 3 R_{E}\right)$, reported a poleward shift of the mean cusp position with a slope of $0.16^{\circ}$ of magnetic latitude per $1^{\circ}$ of tilt. This result confirms the trends observed at lower altitudes.

\subsection{Influence of IMF components on the cusp location}

The location of cusp precipitation and the direction of cuspregion plasma flow strongly depend on the orientation of the interplanetary magnetic field (IMF). Particle measurements in low altitudes have shown that the cusp precipitation shifts: (1) equatorward during intervals of IMF $B_{Z}<0 \mathrm{nT}$ (Newell and Meng, 1989); (2) toward dawn for $B_{Y}<0 \mathrm{nT}$ $\left(B_{Y}>0 \mathrm{nT}\right)$ in the Northern (Southern) Hemisphere (Newell et al., 1989); and (3) toward dusk for $B_{Y}>0 \mathrm{nT}\left(B_{Y}<0 \mathrm{nT}\right)$ in the Northern (Southern) Hemisphere. The $B_{Y}$ shift is more apparent for $B_{Z}<0 \mathrm{nT}$ than $B_{Z}>0 \mathrm{nT}$. The direction of cusp plasma flow is predominantly anti-sunward (sunward) for $B_{Z}<0 \mathrm{nT}\left(B_{Z}>0 \mathrm{nT}\right)$ in the Northern (Southern) Hemisphere (Weiss et al., 1995). During southward IMF, the cusp local magnetic time extent can reach $3.7 \mathrm{~h}$ of MLT. It suggests that the merging process involves the whole dayside magnetopause (Maynard et al., 1997).

The latitudinal width of the cusp in low altitudes is about $1^{\circ}$, somewhat wider for northward $B_{Z}$, and somewhat narrower for southward $B_{Z}$ (Newell and Meng, 1987). For increasingly northward $B_{Z}$, the cusp displays only a slight tendency to move to higher latitudes (Newell et al., 1989).

In higher altitudes $\left(\sim 2 R_{E}\right)$, Aparicio et al. (1991) have been able to distinguish a well-defined region with an average width of $\sim 2^{\circ}$ in invariant latitude of a magnetosheath type of particle precipitation with energies typically less than $200 \mathrm{eV}$ for electrons and $1000 \mathrm{eV}$ for ions. In these altitudes, the plasma, which appeared to have its source in the cusp, was found in a region where the geomagnetic field lines were inferred to be closed, possibly $3^{\circ}-5^{\circ}$ equatorward of the region of open field lines (Potemra et al., 1992). They concluded that this effect is transient in nature and that the solar wind/cusp plasma enters closed geomagnetic field lines by a process associated with a time-varying magnetospheric configuration consisting of a compression and equatorward shift of the cusp, followed by a shift in the cusp to a position associated with northward IMF.

Woch and Lundin (1992) presented the magnetosheath plasma precipitation in the polar cusp and its control by IMF based on the Viking measurements. The azimuthal motion of accelerated magnetosheath plasma and the location of its entry in local time depends on IMF $B_{Y}$ and thus, their observation agrees with the observed shift in the cusp precipitation region toward dawn (dusk) for IMF $B_{Y}$ negative (positive) as it was referred to in low altitudes (Newell et al., 1989). The location in latitude (the equatorward or poleward boundary of the cusp) is controlled by IMF $B_{Z}$. For southward IMF, regions of accelerated plasma are located at the equatorward edge of the cusp, i.e. in the dayside merging region, and for northward IMF, the acceleration site is at the poleward edge where, according to the antiparallel merging hypothesis (Crooker, 1979), merging of the IMF and plasma mantle field can occur.

Zhou et al. (2000) used the Polar magnetic and plasma measurements to determine the cusp position in radial distances between 5 and $9 R_{E}$. They found statistically the cusp 
to be usually from 08:00 to 16:00 MLT. The invariant latitude of the center of the cusp extends from $70^{\circ}$ to $86^{\circ}$ and moves further equatorward for stronger southward IMF. They saw a clear local time shift due to IMF $B_{Y}$ only for southward IMF conditions. The observed local time shift (for $B_{Z}<0 \mathrm{nT}$ ) was consistent with the low-altitude observations (Newell et al., 1989). Slopes of a dependence on IMF $B_{Z}$ seen at both low and high altitudes (DMSP and Polar, Newell et al., 1989; Zhou et al., 2000) are very similar, but low-altitude observations of the cusp are mapped at $\sim 1.5^{\circ}$ equatorward of highaltitude observations. Zhou et al. (2000) attributed these differences to the use of hourly average IMF in the low-altitude study, in contrast to the one-minute average IMF used in their study.

Eastman et al. (2000) studied the Hawkeye data and reported only weak IMF dependencies of the cusp position. For the negative IMF $B_{Z}$ intervals, the cusp was located $3^{\circ}$ lower in invariant latitude than for the positive IMF $B_{Z}$ intervals. This fact is consistent with the low-altitude observations by DMSP (Newell et al., 1989). However, they observed no cusp shift in local time.

The IMF $B_{X}$ effect on the cusp latitudinal position is still ambiguous (e.g. Cowley, 1981; Newell et al., 1989).

\subsection{The effect of solar wind dynamic pressure}

A compression of the magnetosphere by a solar wind pressure enhancement changes the shape of the magnetopause and thus, it can influence the cusp profile and position. Newell and Meng (1994) reported a much wider statistical cusp for high solar wind pressure conditions. They found $4 \mathrm{~h}$ of MLT width for the high-density solar wind with pressures $>4 \mathrm{nPa}$.

Regarding the effects of solar wind dynamic pressure enhancement on the high-altitude polar cusp locations, Zhou et al. (2000) showed that it thickens the cusp in the latitudinal direction and also widens it in magnetic local time. The former result is consistent with that reported by Farrell and Van Allen (1990) using the Hawkeye data. However, the latter result (also based on the Hawkeye observations) appears to be at odds with that reported by Fung et al. (1997), that higher solar wind dynamic pressure shrinks the cusp extent in magnetic local time. Another study based again on the Hawkeye data (Eastman et al., 2000) showed that the cusp moves equatorward for higher values of solar wind dynamic pressures, if the Earth's dipole tilts toward the Sun, and reported an opposite cusp shift, if the dipole axis tilts away from the Sun. However, the second result is not very reliable due to a small number of cusp crossings under these conditions.

The first Interball- 1 and Magion- 4 cusp observations were reported by Sandahl et al. (1997). The authors found the cusp well defined and persistent at all altitudes from $4 R_{E}$ to $13 R_{E}$. We present the statistics of the Magion-4 cusplike plasma observations in the same range of altitudes. The statistics are oriented on the influence of the IMF direction on the location of the high-altitude cusp. The other external
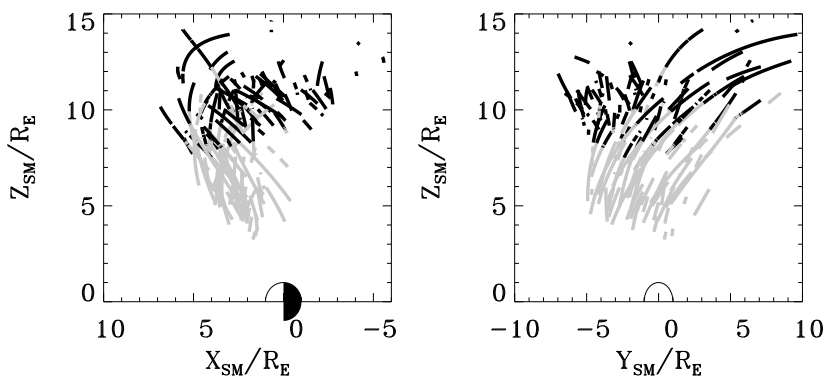

Fig. 1. Cusp crossings observed by Magion-4 during 1995-1997 years plotted in SM coordinates. Different colours distinguish measurements made closer or farther than $3 R_{E}$ from the magnetopause (T96 model).

parameters, such as solar wind dynamic pressure or velocity of the solar wind, are touched on only briefly.

\section{The data set}

Our study is based on particle precipitation patterns observed by the Magion-4 satellite (a part of the Interball project) during the years 1995-1997 (Němeček et al., 1997). We have taken advantage of its orbit which scans the vertical profile of the cusp from an altitude $\sim 3 R_{E}$ up to the magnetosheath. To compare our observations with the low-altitude cusp determination, we have projected all parts of the orbits, on which the cusp-like plasma have been observed, onto the Earth's surface, using the Tsyganenko and Stern (1996) model (T96) of the magnetospheric magnetic field. Our studies of multispacecraft observations of cusp-like plasma confirm the usability of the T96 model to compare cusp observations in different altitudes (Merka et al., 1997).

The T96 model includes four parameters: the solar wind dynamic pressure, $D_{\mathrm{ST}}$ index, the $B_{Y}$ and $B_{Z}$ components of IMF. As a solar wind monitor, we have used the Wind plasma and magnetic field data, shifted by the time of solar wind propagation. The internal state of the magnetosphere was described by an hourly averaged $D_{\mathrm{ST}}$ index.

Our cusp plasma classification is based on the observation of the differential energy flux, which should peak at energy lower than $200 \mathrm{eV}$ for electrons and $1 \mathrm{keV}$ for ions. The threshold for the ion energy flux was $10^{10} \mathrm{eV} /\left(\mathrm{cm}^{2} \mathrm{~s} \mathrm{sr}\right)$. Our criteria are consistent with criteria of the other authors (e.g. Aparicio et al., 1991; Eastman et al., 2000; Newell et al., 1989; Zhou et al.,1999). To exclude the magnetosheath population, we have applied the additional restriction on the bulk velocity to be less than $100 \mathrm{~km} / \mathrm{s}$ or field-aligned. The inbound parts of the orbits crossed the magnetopause near the equatorial plane, but the search for the cusp-like events on outbound parts of the orbits excludes any possible identification of a low-latitude part of the entry layer as a cusp crossing. We will discuss the criteria for the cusp determination later.

All parts of the orbits on which the cusp-like plasma was detected are depicted in Fig. 1 in a solar-magnetic (SM) co- 
ordinate system. The left panel in Fig. 1 presents a typical funnel-shaped cusp with good latitudinal coverage above $\sim 5 R_{E}$. However, a specific feature of Magion-4 is that it was facilitated by a real-time telemetry system which provides high-time resolution measurements, but only during periods of direct visibility of the satellite from the receiving station. It causes a lack of the low-altitude (below $\sim 7 R_{E}$ ) cusp observations in the post-noon sector, which can be seen in the right panel of Fig. 1. If analyzing the high-altitude cusp, a more important factor than the altitude itself is the relative position of measurements with respect to the magnetopause. For this reason, we have computed the distance of the observational point from the model magnetopause (T96 model) and divided our data set into two parts: measurements made closer or farther than $3 R_{E}$ from the magnetopause. These two groups are distinguished by different colours in Fig. 1.

For further processing, we have taken one minute of the measurements as a unit and mapped the position of the spacecraft toward the Earth's surface. The total time of the cusp observations was $\sim 8500 \mathrm{~min}$, but only $\sim 8100 \mathrm{~min}$ of our measurements could be mapped toward the Earth due to resting observations laid out of the model magnetopause. The longest continuous cusp observation lasted 433 min (intervals shorter than 5 min were not considered).

The principal effect influencing the high-altitude cusp location is the dependence on the dipole tilt angle (Newell and Meng, 1989; Zhou et al., 1999; Němeček et al., 2000). As we noted in the Introduction, our analysis of the Magion-4 data (Němeček et al., 2000) revealed the shift in the cusp as high as $0.16^{\circ}$ of magnetic latitude per $1^{\circ}$ of tilt. We have enlarged the data set used in this study by adding the last 3 months of Magion-4 observations, and reanalyzed this dependence. The best fit has a lower slope $\left(0.14^{\circ}\right)$. To account for this fact, we have shifted the latitude of each footprint linearly according to:

$\lambda^{\mathrm{MLAT}}=\lambda_{0}^{\mathrm{MLAT}}-0.14 \vartheta$,

where $\lambda_{0}^{\mathrm{MLAT}}$ and $\lambda^{\mathrm{MLAT}}$ are the original and corrected magnetic latitudes of the cusp projection to the Earth, and $\vartheta$ stands for the dipole tilt angle. Figure 2 demonstrates an influence of the correction on the observations. The depicted quantity represents a number of minutes of cusp observations in bins which are $0.5 \mathrm{~h}$ of MLT long and $1^{\circ}$ of MLAT wide. The top part of the figure shows the footprints of cusp-like plasma observations in original coordinates, and the bottom part shows the same data after our tilt correction. The corrected plot is more compact, with the extreme cusp positions shifted toward the center. We use these corrected footprint positions in the following presentations of our study.

\section{Experimental results}

To take into account the coverage of the regions under question with the Magion- 4 data, we have computed the footprints of all parts of the orbits which lay below the magne-
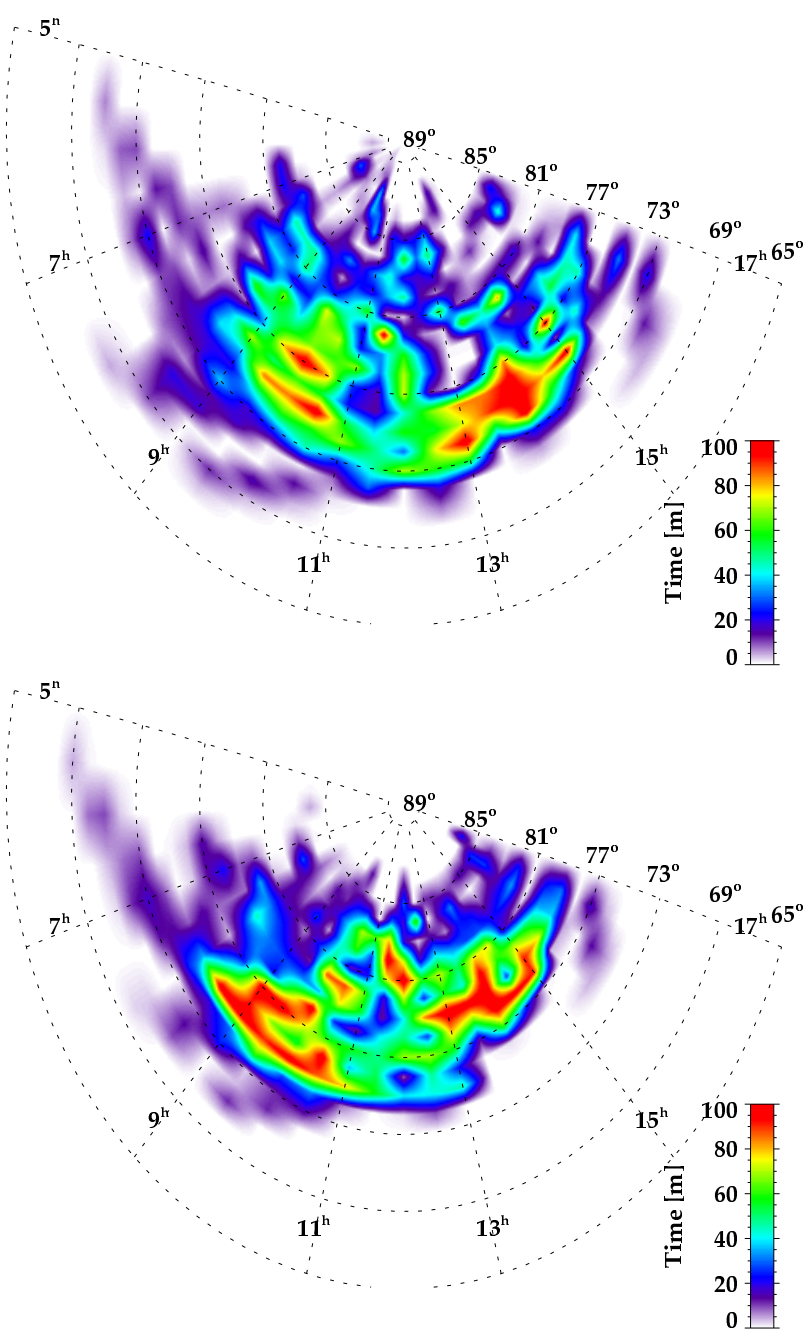

Fig. 2. The number of minutes of cusp-like plasma observations in $0.5 \mathrm{~h} \times 1^{\circ}($ MLT $\times$ MLAT) bins plotted in original (top) and corrected (bottom) coordinates.

topause and on which the ion energy spectra and magnetic field were measured. These footprints were divided into the same bins as our cusp observations. The number of minutes which the satellite spent in the particular bin was counted. The coverage is seen in the top part of Fig. 3. The time of the cusp-like plasma observations (see Fig. 2) was divided by the total time of the observations, in order to obtain the probability of the cusp observations. The resulting plot in the bottom part of Fig. 3 shows that the cusp-like plasma was observed in a large interval in both MLAT and MLT $\left(\sim 70^{\circ}-\right.$ $85^{\circ}$ MLAT and $6.5-16.5 \mathrm{~h}$ MLT). It would be noted that no plasma meeting our cusp definition was observed outside of the area covered by Fig. 3. A hatched area distinguishes those bins crossed by Magion- 4 less than ten times. The cusp-like plasma was measured at $73^{\circ}-83^{\circ}$ MLAT and 7.0 $15.5 \mathrm{~h}$ MLT with a probability greater than $25 \%$ (we consider only non-hatched bins). The maximum of the observation probability is about $50 \%$. This suggests that the cusp is narrow and that its displacement, caused by the different 

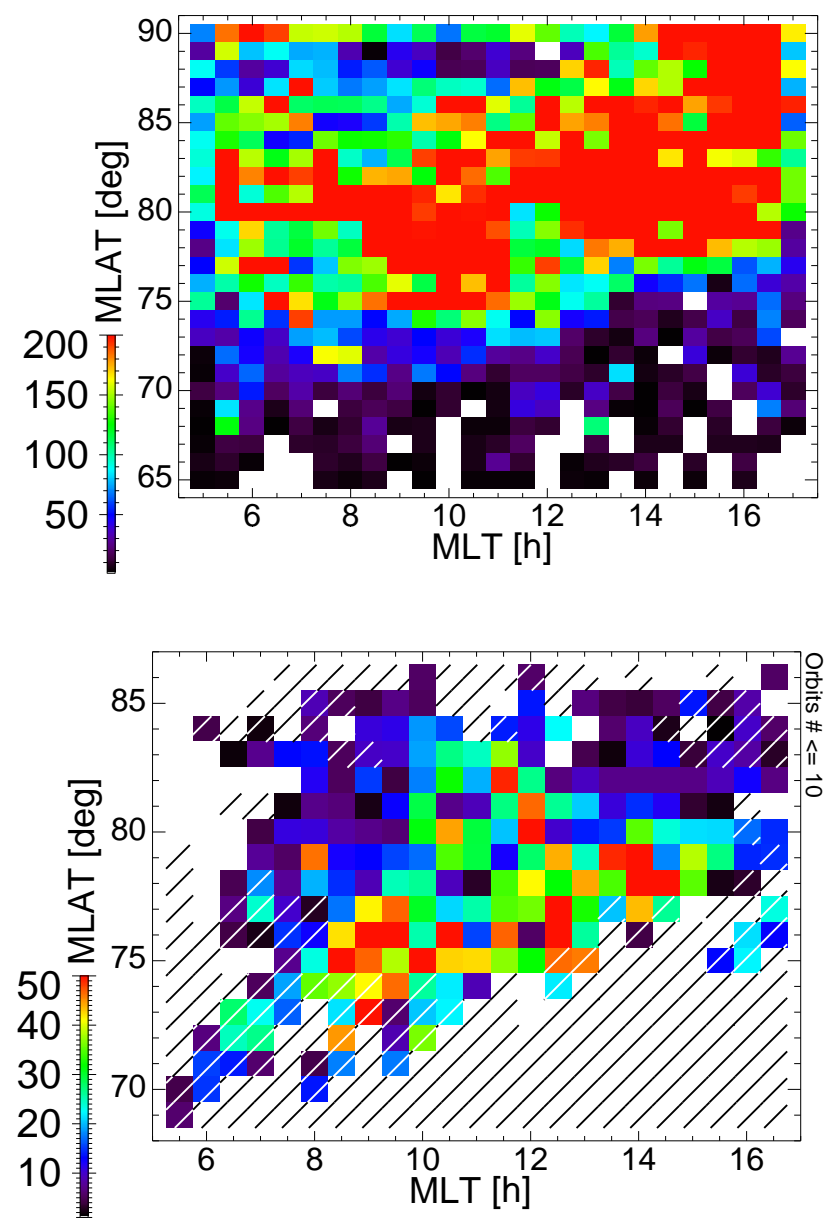

Fig. 3. The total observational coverage of the dayside auroral zone by the Magion- 4 measurements mapped along magnetic field lines down to the Earth's surface (top) and the ratio between the time of the cusp-like plasma observation and the total time of observation (bottom). Magion-4 passed not more than ten times through the hatched bins.

factors (solar wind dynamic pressure, the orientation of IMF, etc.), is higher than the cusp width itself. Based on this interpretation, the cusp latitudinal width can be estimated at $\sim 5^{\circ}-6^{\circ}$ MLAT. The equatorward cusp boundary is located more southward in the pre-noon sector. This could be caused by the dawn-dusk asymmetry of precipitation patterns or by the lower observational coverage in lower latitudes of the post-noon sector (see a top part of Fig. 3 and hatched areas). However, the probability peaks in $75^{\circ}$ MLAT at $\sim 8 \mathrm{~h}$ of MLT and in $78^{\circ}$ MLAT at $\sim 15 \mathrm{~h}$ of MLT. These observations confirm the trend seen for the equatorward boundary and thus, the influence of the data coverage can be ruled out.

The other interesting feature which can be seen in Fig. 3 is that although the non-negligible probability of the cusp observations is concentrated into narrow latitudinal bands $(\sim$ $3^{\circ}$ MLAT) in the morning and afternoon sectors, it is spread nearly equally through all latitudes covered by observations around the local noon.
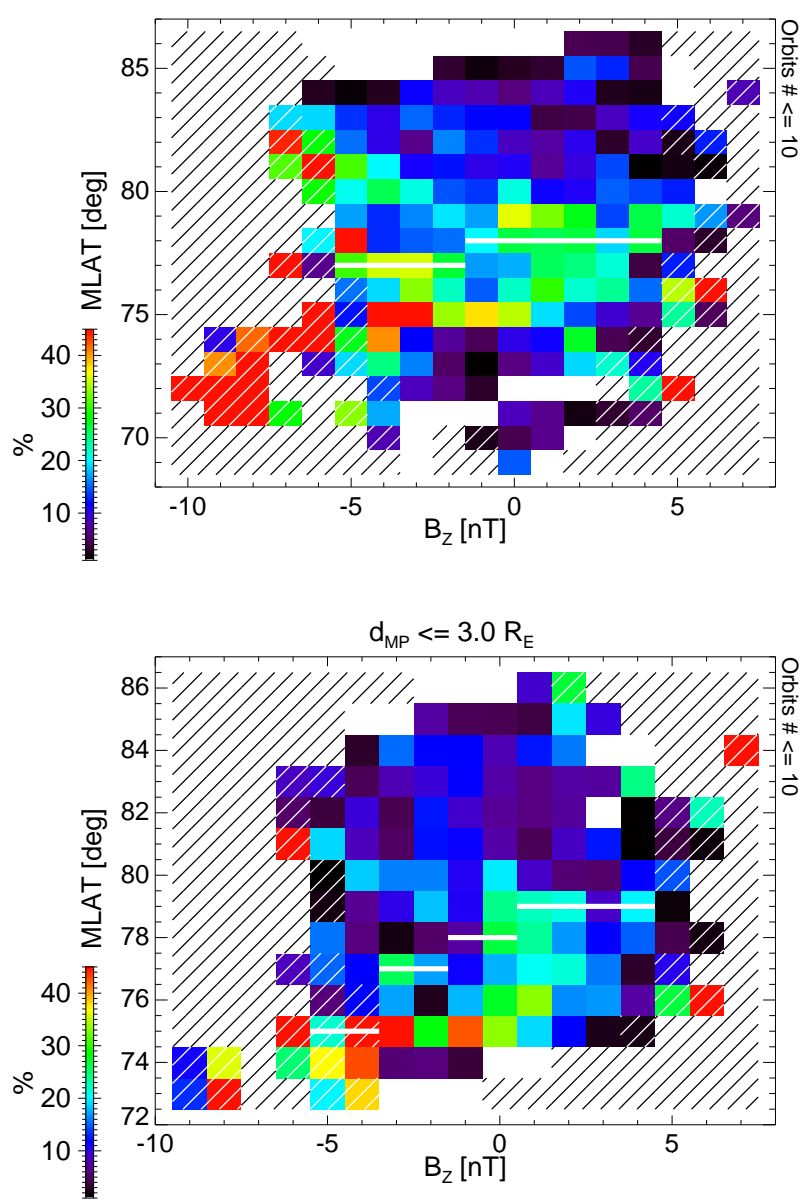

Fig. 4. The cusp location as a function of the IMF $B_{Z}$ component. The bottom part presents cusp observations near the model magnetopause $\left(d_{\mathrm{MP}} \leq 3 R_{E}\right)$.

\subsection{The IMF dependence}

The poleward shift of the cusp position with an increase in the IMF $B_{Z}$ component was well established in lower altitudes. Figure 4 shows the cusp position as a function of the IMF $B_{Z}$ component in high altitudes. The top part presents the whole data set from $\sim 4 R_{E}$ of altitude up to the magnetopause. The white line depicts the median of the probability of the cusp observation. This line shows the poleward shift in the cusp on $1^{\circ}$ MLAT, if IMF $B_{Z}$ changes from -5 to $+5 \mathrm{nT}$. The shift seems to be rather small. However, if we follow the colour code, we can see that the probability peaks in more than one latitude for negative $B_{Z}$. While the peak at $\sim 75^{\circ}$ MLAT and $-5 \mathrm{nT}$ shifts poleward if $B_{Z}$ becomes more positive, the peak at $\sim 80^{\circ}$ MLAT and $-5 \mathrm{nT}$ undergoes an opposite shift and gradually vanishes. These features suggest either a broad cusp, together with an insufficient number of observations for negative $B_{Z}$, or two sources of observed plasma. We will discuss these two possibilities later.

The bottom part in Fig. 4 shows the measurements in the 


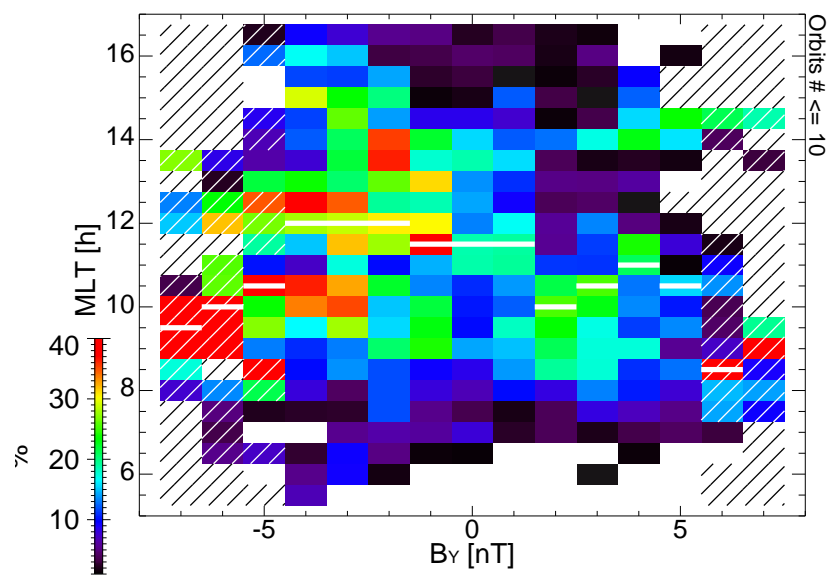

Fig. 5. The cusp longitudinal location as a function of the IMF $B_{Y}$ component.

vicinity of the magnetopause only. For IMF $B_{Z}<0 \mathrm{nT}$, if IMF is more negative, the cusp moves more equatorward (on $\sim 0.5^{\circ}$ per $1 \mathrm{nT}$ ), but when IMF $B_{Z}>0 \mathrm{nT}$, the cusp latitude is approximately constant. These Magion- 4 results on cusp shift with IMF $B_{Z}$ are consistent with results obtained from DMSP at low altitudes (Newell et al., 1989), from Viking at middle altitudes (Woch and Lundin, 1992), and from Polar and Hawkeye at high altitudes (Eastman et al., 2000; Zhou et al., 2000).

In high-latitudes $\left(82^{\circ}-86^{\circ} \mathrm{MLAT}\right)$, we observe an increased rate of the cusp-like plasma observation for northward IMF. Magnetosheath plasma enters these latitudes as a result of the reconnection process northward of the cusp, as shown in Šafránková et al. (1998). Two-point observations (Šafránková et al., 2000) reveal that a steady reconnection takes place tailward of the cusp under this condition and the position of the reconnection site moves in accordance with the actual IMF direction. We suppose that this reconnection is a source of the secondary peak that we can see in Fig. 4.

The influence of the IMF $B_{Y}$ component on the highaltitude cusp position is demonstrated in Fig. 5. For $B_{Y}=$ $-5 \mathrm{nT}$, the median cusp position lies at 1030 MLT and for $B_{Y}=-1 \mathrm{nT}$, at $1130 \mathrm{MLT}$. For positive $B_{Y}$, there is no significant trend of the cusp movement in the median depicted as a white line. This finding is consistent with the results in Eastman et al. (2000). However, the situation is more complicated if the colour-coded probabilities are analyzed. One can identify the cusp population which has a maximum of probability at $\sim 9.5$ MLT and $-7 \mathrm{nT}$. This displacement is fully consistent with expectations for high-latitude reconnection at the Northern Hemisphere. As an addition, we can find another two maxima of probability: at $\sim 13 \mathrm{MLT},-7 \mathrm{nT}$ and 9 MLT, $+6 \mathrm{nT}$. The dawnward shift with increasing $B_{Y}$ suggests that a source of this population is high-latitude reconnection in the Southern Hemisphere.

To compare our result with investigations based on the Polar data (Zhou et al., 2000), we used their magnetic local time
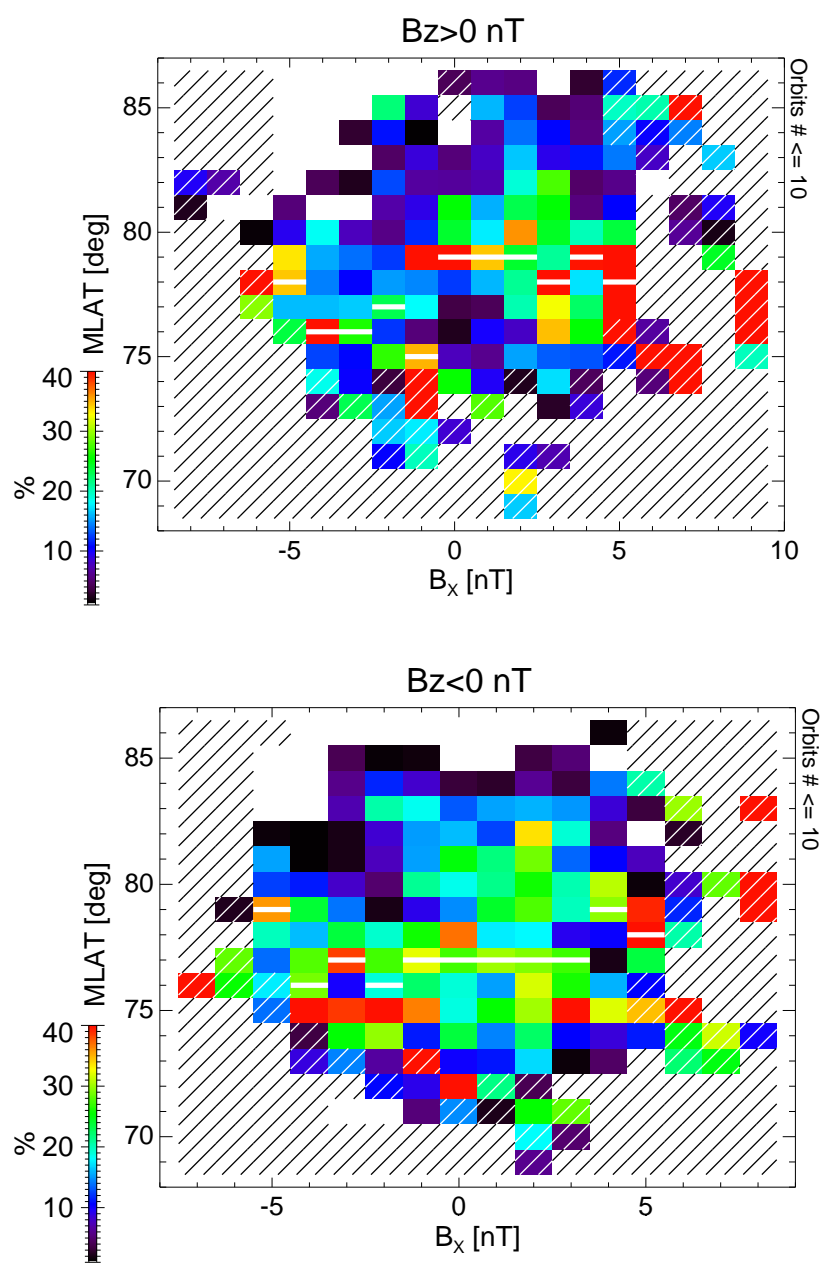

Fig. 6. The cusp latitudinal location as a function of the IMF $B_{X}$ component for $B_{Z}>0 \mathrm{nT}$ (top) and $B_{Z}<0 \mathrm{nT}$ (bottom).

definition. They obtained MLT as a difference in magnetic longitude of the northern cusp foot print and that of the subsolar point. In this case, the cusp movement is not so significant for negative IMF $B_{Y}$, but we found similar dependence for positive values of $B_{Y}$.

Regardless of the MLT definition, our observation indicates a presence of two separated statistical cusp populations for $B_{Y}>0 \mathrm{nT}$. The first peak of probability of cusp observations lies in the pre-noon sector and the second lies in the afternoon sector. According to Zhou et al. (2000), a center of the reconnection site moves duskward in the Northern Hemisphere and dawnward in the Southern Hemisphere for positive $B_{Y}$. Since each reconnection site is connected to both Northern and Southern Hemispheres, this mechanism might be expected to split the cusp and to move two parts away from noon. Furthermore, it could even explain two fuzzy peaks of probability of cusp observations, if the IMF $B_{Y}$ component is positive.

The influence of the IMF $B_{X}$ component on the cusp location has not been found in low altitudes and thus, one can 

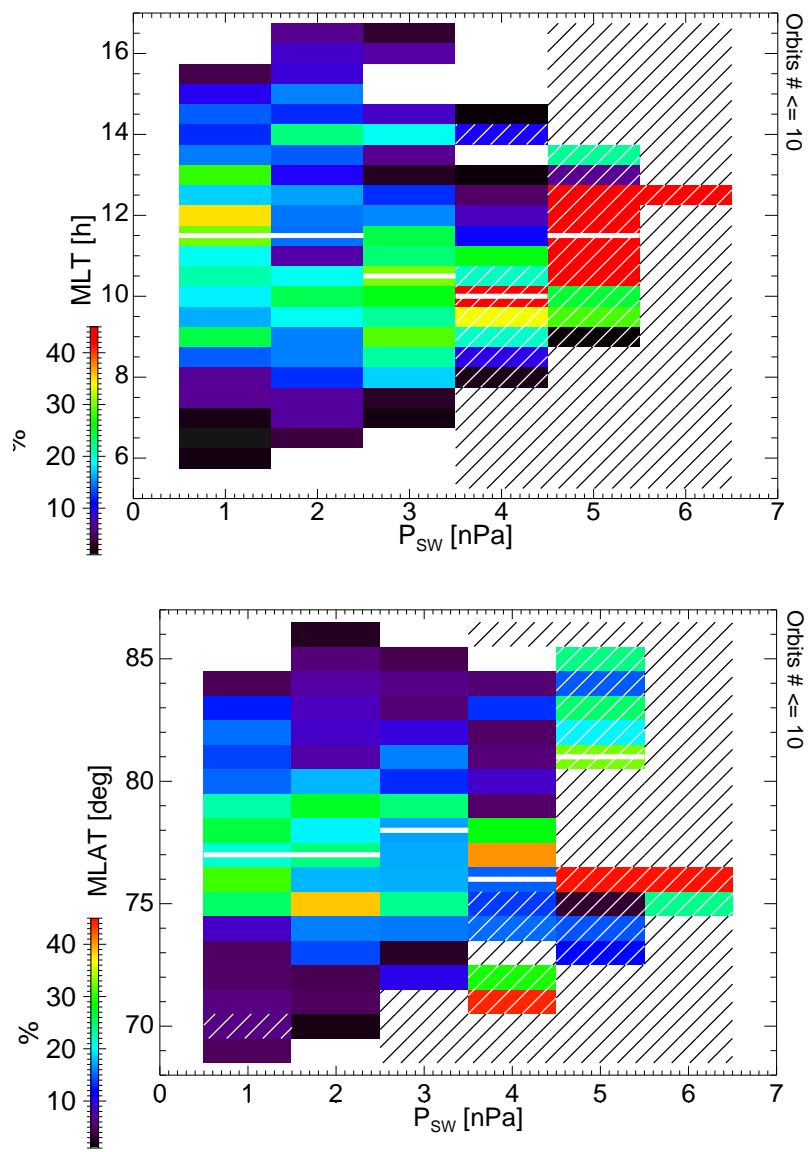

Fig. 7. The longitudinal (top) and latitudinal (bottom) cusp location as a function of the solar wind dynamic pressure.

expect that it would be very weak (if any) in high altitudes. Since the $B_{Z}$ effect is rather strong and could mask other dependencies, we have plotted the latitude of the cusp observation as a function of $B_{X}$ for positive and negative $B_{Z}$ separately in Fig. 6. The bars indicating the median probability do not show any clear $B_{X}$ dependence, but in colours one can again identify two populations which are generally moving equatorward with increasing $B_{X}$. We assume that these two populations are generated at opposite hemispheres during periods of significant $B_{Y}$ component. Their equatorward shift is consistent with the change in the IMF direction due to the drapping effect which converts the positive $B_{X}$ into negative $B_{Z}$ in the Northern Hemisphere.

\subsection{Influence of solar wind parameters}

The low-latitude observations have shown a broader cusp during periods of enhanced solar wind dynamic pressure. Our analysis in Fig. 7 seems to reveal a different behaviour in both longitude (top panel) and latitude (bottom panel). This discrepancy is only apparent because our plots depict the probability of cusp observations. The broader region occupied by non-zero probability means the probably of a higher

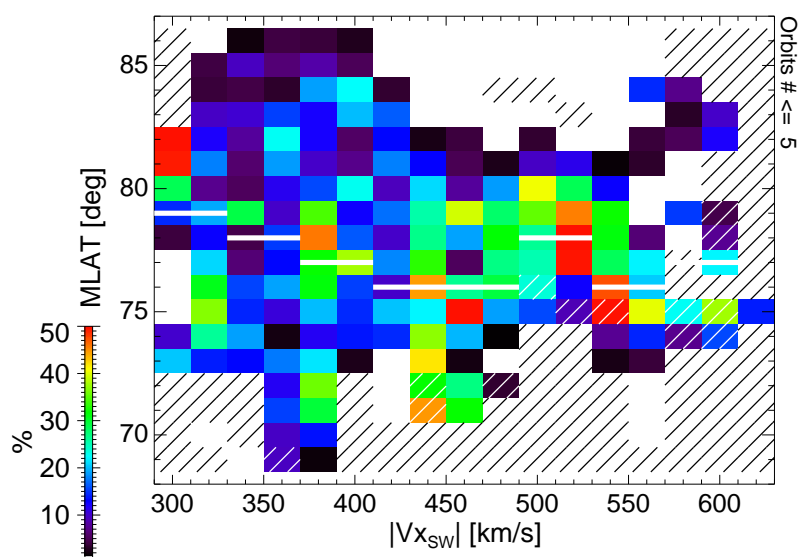

Fig. 8. The cusp latitudinal location as a function of the solar wind velocity $v_{X}$ component.

variability of the cusp position rather than a broader cusp.

A factor which can enhance the variability of the cusp location is the level of magnetosheath fluctuations. Since simultaneous magnetosheath measurements are not available, we have analyzed the cusp latitude as a function of solar wind speed. Figure 8 shows clearly two regions: very large latitudinal spread of the cusp observations with no clear peak of probability for low-speed solar wind $\left(v_{S W} \leq 420 \mathrm{~km} / \mathrm{s}\right)$ and a narrower cusp region for higher speeds. This result is rather surprising because Němeček et al. (2000) show that a level of magnetosheath fluctuations increases with increasing solar wind speed. However, there can be other factors causing the broadening of the region where the cusp can be observed as the variability of the interplanetary magnetic field or KelvinHelmholtz instability. The correlation of these factors with solar wind speed was never experimentally investigated.

There is a slight trend of an equatorward shift of the cusp with increasing solar wind speed. This trend is consistent with the positive correlation of the solar wind speed and geomagnetic activity. We have checked this correlation in our data and found a linearly increasing $\left|D_{S T}\right|$ index with the speed. Thus, the observed changes in the cusp location are consistent with the expansion of the auroral oval during geomagnetically disturbed periods (Meng, 1984).

\section{Summary and discussion}

The behaviour of the plasma and magnetic field in the highaltitude cusp strongly differs from those observed in lower altitudes because the high altitude cusp represents an interface between two regimes. The energy connected with the plasma bulk flow dominates in the magnetosheath, whereas the Earth's magnetic field determines the plasma properties in lower altitudes. The ion and electron distributions undergo significant evolution on the path from the magnetosheath to the ionosphere due to the time-of-flight effect (Lockwood et 
Table 1. Mean and median cusp positions near the magnetopause $\left(d_{\mathrm{MP}} \leq 3 R_{E}\right)$

\begin{tabular}{lccccr}
\hline Criteria & \multicolumn{2}{c}{ MLAT $\left[{ }^{\circ}\right]$} & \multicolumn{2}{c}{ MLT [h] } & Obs. \\
& $\langle\lambda\rangle \pm \sigma$ & $M_{\lambda}$ & $\langle l\rangle \pm \sigma$ & $M_{l}$ & {$[\mathrm{~min}]$} \\
\hline All & $79.0 \pm 2.9$ & 79.0 & $11.7 \pm 2.3$ & 11.9 & 3984 \\
$B_{X}<0 \mathrm{nT}$ & $78.3 \pm 3.0$ & 77.4 & $11.4 \pm 2.3$ & 11.4 & 1601 \\
$B_{X}>0 \mathrm{nT}$ & $79.5 \pm 2.7$ & 79.4 & $11.8 \pm 2.3$ & 12.0 & 2360 \\
$B_{Y}<0 \mathrm{nT}$ & $79.1 \pm 2.8$ & 79.2 & $11.8 \pm 2.0$ & 12.1 & 2374 \\
$B_{Y}<0 \mathrm{nT}$ & & & & & \\
$\& B_{Z}<0 \mathrm{nT}$ & $78.9 \pm 2.8$ & 79.4 & $11.5 \pm 1.9$ & 11.6 & 1354 \\
$B_{Y}<0 \mathrm{nT}$ & & & & & \\
$\& B_{Z}>0 \mathrm{nT}$ & $79.5 \pm 2.7$ & 79.1 & $12.3 \pm 1.9$ & 12.8 & 966 \\
$B_{Y}>0 \mathrm{nT}$ & $78.8 \pm 3.0$ & 78.2 & $11.4 \pm 2.6$ & 11.1 & 1597 \\
$B_{Y}>0 \mathrm{nT}$ & & & & & \\
$\& B_{Z}<0 \mathrm{nT}$ & $78.5 \pm 3.4$ & 77.9 & $11.4 \pm 2.5$ & 11.1 & 711 \\
$B_{Y}>0 \mathrm{nT}$ & & & & & \\
$\& B_{Z}>0 \mathrm{nT}$ & $79.0 \pm 2.6$ & 78.6 & $11.5 \pm 2.7$ & 11.1 & 850 \\
$B_{Z}<0 \mathrm{nT}$ & $78.7 \pm 3.0$ & 79.1 & $11.4 \pm 2.2$ & 11.6 & 2069 \\
$B_{Z}>0 \mathrm{nT}$ & $79.3 \pm 2.7$ & 78.9 & $11.9 \pm 2.4$ & 12.2 & 1825 \\
$p_{\mathrm{SW}} \leq 3 \mathrm{nPa}$ & $79.0 \pm 2.9$ & 79.0 & $11.6 \pm 2.3$ & 11.8 & 3766 \\
$p_{\mathrm{SW}}>3 \mathrm{nPa}$ & $79.0 \pm 3.1$ & 78.8 & $12.3 \pm 1.5$ & 12.4 & 218 \\
$\left|v_{x}\right|>450 \mathrm{~km} / \mathrm{s}$ & $77.8 \pm 2.4$ & 77.9 & $12.7 \pm 2.0$ & 13.0 & 1125 \\
$\left|v_{x}\right| \leq 450 \mathrm{~km} / \mathrm{s}$ & $79.5 \pm 2.9$ & 79.3 & $11.3 \pm 2.3$ & 11.2 & 2859 \\
$D_{\mathrm{ST}}<-20 \mathrm{nT}$ & $78.0 \pm 2.9$ & 77.6 & $11.2 \pm 2.0$ & 10.8 & 1025 \\
$D_{\mathrm{ST}} \geq-20 \mathrm{nT}$ & $79.4 \pm 2.8$ & 79.2 & $11.8 \pm 2.3$ & 12.1 & 2959 \\
$K_{P}<3$ & $79.1 \pm 2.8$ & 79.0 & $11.6 \pm 2.4$ & 11.9 & 3299 \\
$K_{P} \geq 3$ & $78.6 \pm 3.1$ & 79.1 & $12.0 \pm 1.8$ & 11.9 & 685 \\
\hline & & & & &
\end{tabular}

al., 1995). The main task of the cusp definition in low and middle altitudes is to distinguish the plasmas of magnetospheric and magnetosheath origin, whereas the distinguishing of the cusp and magnetosheath plasmas is the principal problem in high altitudes near the magnetopause.

In spite of rather strong criteria used for the cusp determination, we have found the cusp-like plasma in broad ranges of latitudes and longitudes. Our limits on peak ion energy and energy flux are the same or harder than those used in Newell and Meng (1988) for the cusp proper. The additional requirement on the velocity to be low or aligned (parallel) to the magnetic field is used to distinguish the plasma mantle where the velocity would be antiparallel in the Northern Hemisphere.

However, the whole region is highly turbulent, and the amplitudes of fluctuations of all parameters exceed their mean values. The waves launching from this region can significantly modify the particle distribution on their path to the ionosphere. The other possible source of such modification can be field-aligned potentials at the cusp boundaries. The evolution of the distribution function would lead to a different classification of the regime of the same plasma in lower altitudes.

We have analyzed the influence of several upstream parameters on the observation probability that the cusp-like precipitation will be observed at a particular point in space. This approach lowers inaccuracies caused by the irregular observational coverage of the region under study and it can even pronounce observed dependencies. However, previously published cusp studies do not usually use any normalization of cusp observations with respect to the overall time of observations.

To compare our results with results published, we calculated mean and median cusp positions based only on the time of cusp-like plasma observations. A survey of calculations in the vicinity of the magnetopause is summarized in Table 1. The first column describes criteria used for data selection, and the next two columns contain cusp positions in MLAT and MLT (mean, standard deviation, and median values).

In low altitudes, the probability of observing the cusp was found to be sharply peaked at $12 \mathrm{~h}$ MLT and the cusp boundaries were found between $74^{\circ}$ and $78^{\circ}$ MLAT. The Magion4 observations have shown a similar mean cusp position at $79.0^{\circ} \pm 2.9^{\circ}$ MLAT and $11.7 \pm 2.3 \mathrm{~h}$ MLT. Viking, Polar, and Hawkeye (e.g. Kremser and Lundin, 1990; Zhou et al., 2000; Eastman et al., 2000) measurements presented a cusp latitudinal position slightly poleward at $\sim 80^{\circ}$ INL. This difference could be caused by the usage of invariant latitude instead of magnetic latitude, because invariant latitude is closely associated with the dipolar field concept, which is not valid near the magnetopause.

Due to a low number of cusp-like plasma measurements during periods of higher solar wind dynamic pressure $(\sim 6 \%$ at $p_{S W}>3 \mathrm{nPa}$ ), we cannot deduce the pressure influence on both the shape and location of the cusp. However, the lower variability of the cusp location during periods of enhanced solar wind pressure suggests that the influence of the IMF orientation on the cusp location is depressed.

In contrast to the other observations, we observe an equatorward movement of the cusp on $\sim 1^{\circ}$ MLAT, if the IMF $B_{X}$ component changes from positive to negative values. A possible explanation was suggested in Cowley (1981), but the shift could be an artificial effect of the T96 model which is not parametrized by IMF $B_{X}$.

In their studies, Newell et al. (1989); Fung et al. (1997); Zhou et al. (2000) found a dawnward cusp movement for $B_{Y}<0 \mathrm{nT}$ and a less distinct duskward shift of the cusp for $B_{Y}>0 \mathrm{nT}$. Although both mean and median cusp positions presented in Table 1 suggest an opposite dependence on IMF $B_{Y}$, we can conclude that our observations agree with the mentioned papers for $B_{Y}<0 \mathrm{nT}$ (as discussed above). On the other hand, a statistical cusp splits into two parts when the IMF $B_{Y}$ component becomes positive (Fig. 5). In this figure, one can identify two maxima even for $B_{Y}<0$, but their local time separation is shorter.

The mean (median) cusp is located slightly equatorward for $B_{Z}<0 \mathrm{nT}$, which confirms previous observations by the DMSP, Polar and Hawkeye satellites (Newell and Meng, 1987; Eastman et al., 2000; Zhou et al., 2000). We have found that a weak dependence of the cusp location on IMF $B_{Z}$ can be connected with the fact that the plasma mantle can meet our definition of cusp-like plasma. According to a generally accepted scheme, reconnection at the subsolar magnetopause moves the cusp equatorward during intervals 
of negative IMF $B_{Z}$. The entering plasma is reflected at low altitudes and creates a distinct mantle which maps poleward of the cusp proper. During northward IMF, the plasma mantle is very thin or disappears (Rosenbauer et al., 1975). This means that the inspected cusp-like region would be broader during strongly negative IMF $B_{Z}$. This effect is clearly seen in both panels of Fig. 4.

During periods of enhanced geomagnetic activity, which is described by the $D_{\mathrm{ST}}$ and $K_{P}$ indices, both mean and median cusp positions are displaced equatorward. In Table 1, we can see that the shift is the same order for both indices. In addition, this influence correspond to a dependence on the $v_{X}$ component of the solar wind velocity. Our observations suggest that the geomagnetic indices depend almost linearly on $v_{X}$.

The detail analysis of the probability of the cusp observations has shown that the observation of two sources of the cusp precipitation, which were attributed to reconnection in conjugated hemispheres, is a common feature in high altitudes. These two sources are projected onto different MLT and their separation seems to be controlled by IMF $B_{Y}$. This effect can explain a low number (Fig. 2), as well as a lower probability of cusp observations (Figs. 3 and 5) near the local noon, since periods of negligible IMF $B_{Y}$ are rare in the solar wind.

The two cusp population can be separated in latitude probably during periods of negative $B_{Z}$ (Fig. 4) and this fact influences the mean cusp positions given in Table 1. The possibility of the latitudinal separation of two cusp sources were pointed out and documented by DMSP observations by Wing et al. (2000).

The high-altitude cusp occupies a broader region than can be expected from low- and middle-altitude observations (e.g. Aparicio et al., 1991; Newell and Meng, 1994; Maynard et al., 1997). It is valid for latitudinal as well as longitudinal extents of the high-altitude cusp projection. The high-altitude cusp observations by the Hawkeye (Eastman et al., 2000) and Polar (Zhou et al., 2000) satellites show a little bit narrower cusp than our study, but the difference is quite small (a few degrees of MLAT and $\sim 1 \mathrm{~h}$ of MLT) and could be attributed to different altitudes of observations. We can conclude that the higher altitude of observations results in the broader cusp region.

\section{Conclusion}

For statistical study of the cusp location, we have identified $\sim 8500$ min of cusp-like plasma observations in Magion-4 plasma data which were measured from $\sim 4 R_{E}$ up to the magnetosheath altitudes. The analysis of our results leads to the following conclusions:

- The major parameter affecting overall cusp configuration at high altitudes is the dipole tilt (e.g. Němeček et al., 2000). Near the magnetopause, the cusp moves on $0.14^{\circ}$ MLAT per $1^{\circ}$ of tilt;
- At high altitudes, the cusp occupies a broader region than can be expected from low- and middle-altitude observations (Merka et al., 1999). A comparison with the DMSP, Viking, Polar, and Hawkeye satellite data suggests widening of the cusp at high altitudes. It is valid for latitudinal as well as longitudinal extents of the highaltitude cusp projection;

- Although we observe generally similar trends of the IMF and/or solar wind influences on the cusp locations, as have already been reported, we have found a few differences:

- The cusp position possibly depends on the direction of the IMF $B_{X}$ component;

- For $B_{Y} \neq 0$, the cusp splits into two parts in magnetic local time;

- The probability of cusp observations shrinks in both latitude and longitude during periods of higher solar wind dynamic pressure. This result could be influenced by a low number of cusp-like plasma measurements for $p_{S W}>3 \mathrm{nPa}$.

We should note that our definition of cusp-like plasma is rather simple and the resulting region may involve plasma from different magnetospheric regions (cusp, cleft, and mantle). However, the high-altitude cusp region is highly turbulent; the satellite observes the merging process in situ and not only its products, and thus, it is very difficult to distinguish among the regions as the cusp, cusp proper or cleft. We should note that the cusp region adjacent to the magnetopause was never investigated and that there is no corresponding definition of this region. The division into subregions, such as cusp proper, cleft or plasma mantle, probably requires an evolution of the distribution during precipitation and cannot be done immediately after plasma entry into the magnetosphere.

For this reason, our study collects them into one region occupied by the cusp-like plasma. In contrast to the lowand middle-altitude studies, the main problem is the separation of the cusp-like region and magnetosheath at high altitudes, as demonstrated in (Merka et al., 2000). Nevertheless, the broadness and movements of the region occupied by the cusp-like plasma cannot be explained by this effect and it is likely a result of a complicated interaction between the interplanetary and magnetospheric magnetic fields and partly a result of the magnetospheric model used.

Acknowledgement. The work was supported by the Czech Grant Agency under Contract No. 205/00/1686 and by the Charles University Grant Agency, Contract 168.

Topical Editor G. Chanteur thanks R. Lundin and J. Blecki for their help in evaluating this paper. 


\section{References}

Aparicio, B., Thelin, B., and Lundin R.: The polar cusp from a particle point of view: A statistical study based on Viking data, J. Geophys. Res., 96, 14 023-14 031, 1991.

Boardsen, S. A., Eastman, T. E., Sotirelis, T., and Green, J. L.: An empirical model of the high-latitude magnetopause, J. Geophys. Res., 105, 23 193, 2000.

Cowley, S. W. H.: Asymmetry efeects associated with the $x$ component of the IMF in a magnetically open magnetosphere, Planet. Space Sci., 29, 809-818, 1981.

Crooker, N. U.: Dayside merging and cusp geometry, J. Geophys. Res., 84, 951-959, 1979.

Eastman, T. E., Boardsen, S. A., Chen, S.-H., Fung, S. F., and Kessel, R. L.: Configuration of high-latitude and high-altitude boundary layers, J. Geophys. Res., 105, 23 221, 2000.

Farrell, W. M. and Van Allen, J. A.: Observations of the Earth's polar cleft at large radial distances with the Hawkeye 1 magnetometer, J. Geophys. Res., 95, 20 945-20 958, 1990.

Fung, S. F., Eastman, T. E., Boardsen, S. A., and Chen, S.-H.: Highaltitude cusp positions sampled by the Hawkeye satellite, Phys. Chem. Earth, 22, 653-662, 1997.

Kremser, G. and Lundin, R.: Average spatial distributions of energetic particles in the mid-altitude cusp/cleft region observed by Viking, J. Geophys. Res., 95, 5753-5766, 1990.

Lockwood, M., Davis, D. J., Smith, M. F., Onsager, T. G., and Denig, W. F.: Location and characteristics of the reconnection $X$ line deduced from low-altitude satellite and ground-based observations, 2. Defense Meteorological Satellite Program and European Incoherent Scatter data, J. Geophys. Res., 100, 21803 $21814,1995$.

Lundin, R.: Plasma composition and flow characteristics in the magnetospheric boundary layers connected to the polar cusp, in: The Polar Cusp, (Eds) Holtet, J. A. and Egeland, A., D. Reidel Publ. Co., Dordrecht, Holland, 9, 1985.

Maynard, N. C., Weber, E. J., Weimer, D. R., Moen, J., Onsager, T., Heelis, R. A., and Egeland, A.: How wide in magnetic local time is the cusp? An event study, J. Geophys. Res., 102, 4765-4776, 1997.

Meng, C.-I.: Dynamic variation of the auroral oval during intense magnetick storms, J. Geophys. Res., 89, 227, 1984.

Měrka, J., Šafránková, J., and Němeček, Z.: Topology of the cuspmagnetosheath transition, in: Proceedings of Contribution Papers: Part II - Physics of Plasmas and Ionized Media, (Ed) Šafránková, J., Praha, MFF UK, 197-203, 1997.

Měrka, J., Šafránková, J., and Němeček, Z.: Interball observations of the high-altitude cusp-like plasma: A statistical study, Czech. J. Phys., 49, 4a, 695-709, 1999.

Měrka J., Šafránková, J., Němeček, Z., Savin, S., and Skalsky, A.: High-altitude cusp: Interball observations, Adv. Space Res., 25, 7/8, 1425-1434, 2000.

Němeček, Z., Fedorov, A., Šafránková, J., and Zastenker, G.: Structure of the low-latitude magnetopause: Magion-4 observations, Ann. Geophysicae, 15, 553-561, 1997.

Němeček, Z., Měrka, J., and Šafránková, J.: The tilt angle control of the outer cusp position, Geophys. Res. Lett., 27, 1, 77-80, 2000.

Němeček, Z., Šafránková, J., Zastenker, G. N., and Pisoft P.: Statistical study of ion flux fluctuations in the magnetosheath, Czech.
J. Phys., submitted, 2001.

Newell, P. T. and Meng, C.-I.: Cusp width and $B_{z}$ : Observations and conceptual model, J. Geophys. Res., 92, 13 673-13678, 1987.

Newell, P. T. and Meng, C.-I.: The cusp and the cleft/boundary layer: Low-altitude identification and statistical local time variation, J. Geophys. Res., 93, 14 549-14 556, 1988.

Newell, P. T. and Meng, C.-I.: Dipole tilt angle effects on the latitude of the cusp and cleft/low-latitude boundary layer, J. Geophys. Res., 94, 6949-6953, 1989.

Newell, P. T. and Meng, C.-I.: Ionospheric projections of magnetospheric regions under low and high solar wind pressure conditions, J. Geophys. Res., 99, 273-286, 1994.

Newell, P. T., Meng, C.-I., Sibeck, D. G., and Lepping, R.: Some low-altitude cusp dependencies on the interplanetary magnetic field, J. Geophys. Res., 94, 8921-8927, 1989.

Paschmann, G., Haerendel, G., Sckopke, N., and Rosenbauer, H.: The entry layer, J. Atm. Terr. Phys., 40, 257, 1978.

Potemra, T. A., Erlandson, R. E., Zanetti, L. J., Arnoldy, R. L., Woch, J., and Friis-Christensen, E.: The dynamic cusp, J. Geophys. Res., 97, 2835-2844, 1992.

Rosenbauer, H., Grunwaldt, H., Montgomery, M. D., Paschmann, G., and Sckopke, N.: Heos 2 plasma observations in the distant magnetosphere: The plasma mantle, J. Geophys. Res., 80, 2723, 1975.

Sandahl, I., Lundin, R., Yamauchi, M., Eklund, U., Safrankova, J., Němeček, Z., Kudela, K., Lepping, R. P., Lin, R. P., Lutsenko V. N., and Sauvaud J.-A.: Cusp and boundary layer observations by Interball, Adv. Space Res., 20, 4/5, 823-832, 1997.

Šafránková, J., Němeček, Z., Sibeck, D. G., Přech, L., Měrka, J., and Santolík, O.: Two-point observation of high-latitude reconnection, Geophys. Res. Lett., 25, 23, 4301-4304, 1998.

Šafránková, J., Měrka, J., and Němeček, Z.: Plasma flow across the cusp-magnetosheath boundary under northward IMF, Adv. Space Res., submitted, 2000.

Tsyganenko, N. A. and Stern, D. P.: A new-generation global magnetosphere field model based on spacecraft magnetometer data, ISTP Newsletter, 6/1, 21, 1996.

Weiss, L. A., Reiff, P. H., Weber, E. J., Carlson, H. C., Lockwood, M., and Peterson, W. K.: Flow-aligned jets in the magnetoshperic cusp: Results from the Geospace Environment Modeling Pilot program, J. Geophys. Res., 100, 7649-7659, 1995.

Wing, S., Newell, P. T., and Ruohoniemi, J. M.: Double cusp: a prediction and a confirmation, AGU Fall Meeting, San Francisco, SM62B-06, F1024, 2000.

Woch, J. and Lundin, R.: Signatures of transient boundary layer processes observed with Viking, J. Geophys. Res., 97, 14311447, 1992.

Zhou, X. W. and Russell, C. T.: The location of the high-latitude polar cusp and the shape of the surrounding magnetopause, J. Geophys. Res., 102, 105-110, 1997.

Zhou, X.-W., Russell, C. T., Le, G., Fuselier, S. A., and Scudder, J. D.: The polar cusp location and its dependence on dipole tilt, Geophys. Res. Lett., 26, 429-432, 1999.

Zhou, X. W., Russell, C. T., and Le, G.: Solar wind control of the polar cusp at high altitude, J. Geophys. Res., 105, 245-251, 2000. 